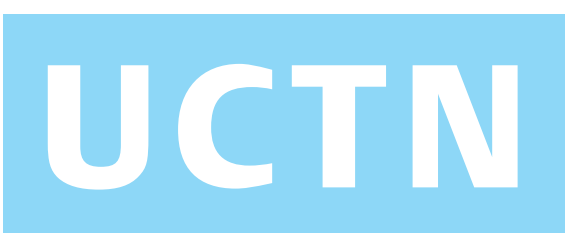

\title{
Technique for Endoscopic Hemostasis of a Bleeding Peptic Ulcer in a Gastrostomy Patient
}

Gastric ulcers induced by gastrostomy are usually located on the posterior wall of the gastric body, and they may result in upper gastrointestinal bleeding [1,2]. A suggested mechanism for the way in which a percutaneous endoscopic gastrostomy (PEG) is capable of inducing gastric ulcers is prolonged contact between the tapered tip of the gastrostomy tube and the posterior wall [3]. The design of the gastrostomy tube has also been reported to be a factor in ulcer bleeding [4]. Esophagogastroduodenoscopy (EGD) is the standard method of establishing the diagnosis, but it involves difficult tangential viewing of gastric ulcers on the posterior wall. We present here a case in which an alternative route was used to manage this problem.

A 62-year-old man with a history of squamous-cell carcinoma of the larynx, who had been treated with long-term percutaneous endoscopic gastrostomy (PEG) feeding, was admitted for massive hematemesis. An EGD was carried out in the usual way, via the oral route under local anesthesia. After evacuation of blood clots from the stomach, a $1.5-\mathrm{cm}$ gastric ulcer with active spurting arterial bleeding (Forrest Ia) was observed on the posterior wall of the gastric body, just opposite the tip of the PEG (Figure 1a). Due to the tangential angulation of the scope, endoscopic hemostasis by epinephrine injection and placement of three Hemoclips was carried out with difficulty. The practice of scheduling a second endoscopy in patients with bleeding peptic ulcers has been adopted in our unit [5], and this was arranged for the following day. The gastric ulcer was still showing spurting arterial bleeding. Only one of the Hemoclips was still loosely attached to the edge of the ulcer. Epinephrine and heater-probe treatment were used for hemostasis.

A third EGD procedure on the following day showed a gastric ulcer with a visible vessel (Forrest IIa). Open surgery is usually considered in this situation, but in this
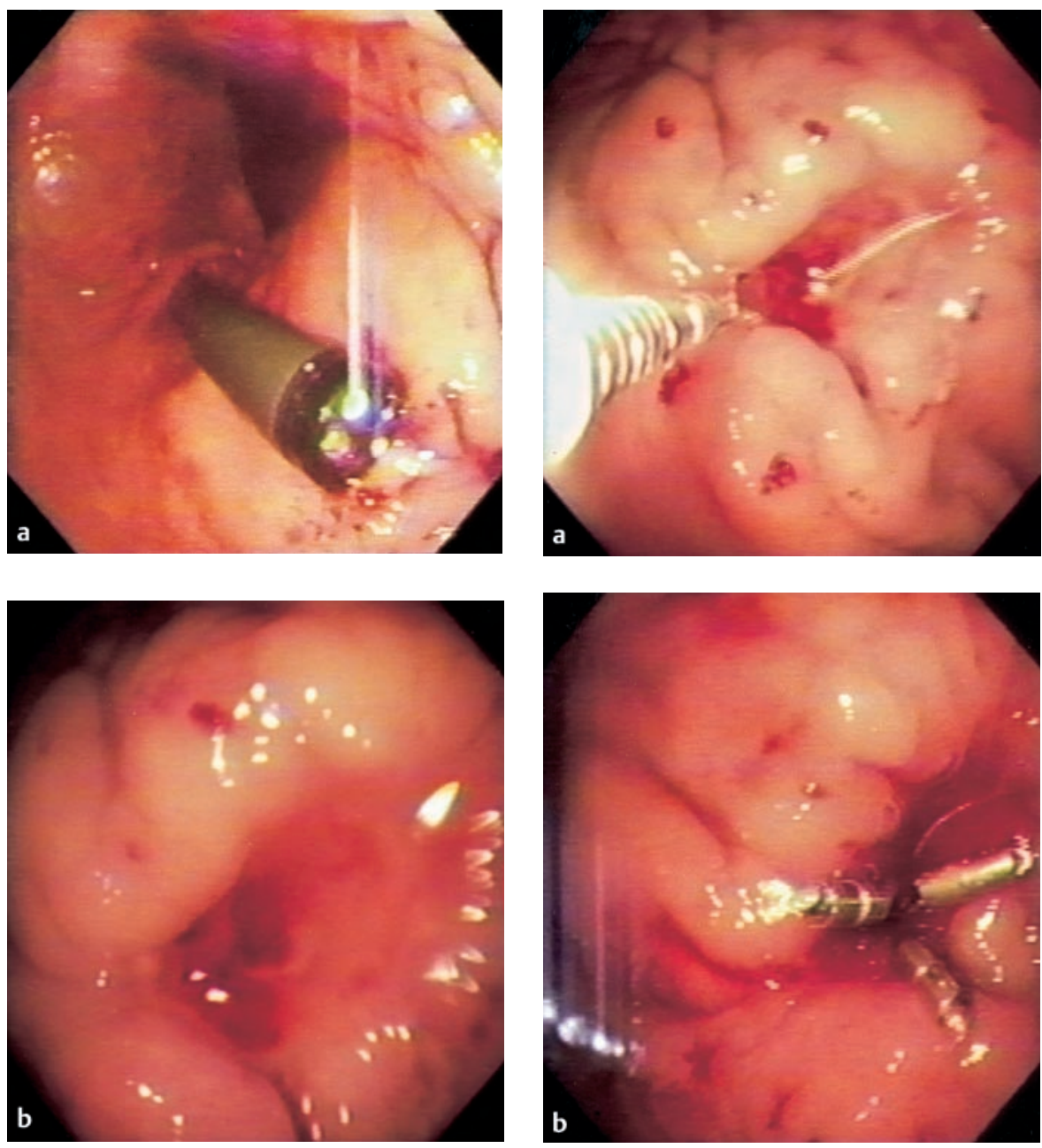

Figure 1 a Esophagogastroscopy revealed a gastric ulcer on the posterior wall of the stomach, just opposite the tip of the gastrostomy tube. A bleeding vessel is seen at the base of the ulcer. Effective endoscopic hemostasis is technically difficult due to the tangential viewing of the ulcer with this approach. b Upper endoscopy via the gastrostomy site provides an end-on view of the bleeding gastric ulcer. Effective endoscopic hemostasis can be achieved with ease.

case the patient was not offered surgery, as we believe that endoscopy via the gastrostomy site is capable of providing endon visualization of the ulcer for effective hemostasis.

Technique. The patient was placed in the supine position, and the PEG tube was removed. An endoscope was inserted into

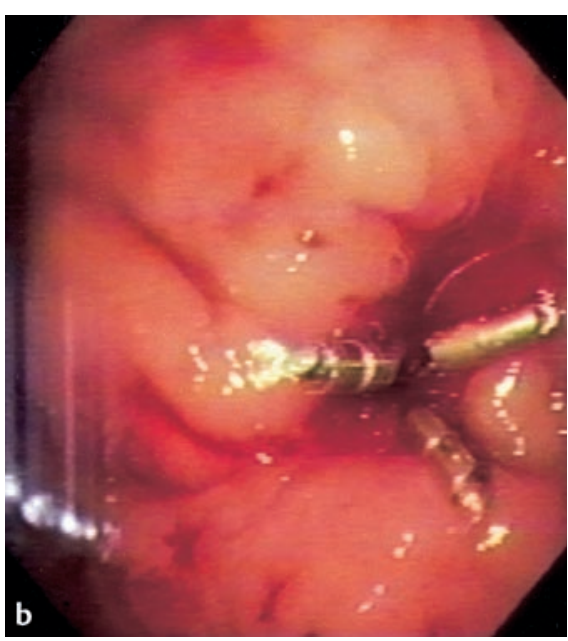

Figure 2 a Successful hemostasis of the bleeding gastric ulcer is achieved after epinephrine injection and the application of four Hemoclips. b On the following day, a fourth endoscopy via the gastrostomy route showed no evidence of recurrent ulcer bleeding. The four Hemoclips were still firmly attached to the base of the ulcer.

the stomach via the PEG site. The ulcer was approached with end-on viewing in this position (Figure $\mathbf{1} \mathbf{b}$ ), and hemostasis was achieved by injecting epinephrine and placing four Hemoclips (Figure 2a). A fourth endoscopic examination via the gastrostomy route the next day showed no evidence of recurrent ulcer bleeding. The four Hemoclips were still firmly attached to the ulcer base (Figure $\mathbf{2} \mathbf{b}$ ). The patient's PEG tube was reinserted, and he 
was treated with an 8-week course of proton-pump inhibitors for ulcer healing.

It is a matter of controversy whether upper endoscopy should be performed routinely via the gastrostomy route, without a conventional EGD via the oral route. The alternative approach described here may involve less discomfort for the patient, and it allows effective treatment of PEG-induced posterior wall ulcers. However, bleeding may not always be related to the PEG in these cases, and esophageal lesions might be missed. It will only be possible to settle this issue as experience with upper endoscopy via the gastrostomy route in these patients accumulates.

\section{Y. Choi, P. W. Y. Chiu, P. K. H. Kwong,} S. H. Lam

Dept. of Surgery, United Christian Hospital, Hong Kong, China.

\section{References}

${ }^{1}$ Delator J, Boylan JJ. Bleeding gastric ulcer: a complication from gastrostomy tube replacement. Gastrointest Endosc 2000; 51: $482-484$

${ }^{2}$ Wiener GJ. Complications caused by the tip of gastrostomy tubes and Foley catheters. Am J Gastroenterol 1999; 94: 3656-3657

${ }^{3}$ Nzeako UC, Murray JA, Chari ST. Role of tube design and selection in occurrence of gastric lesser curvature ulceration by percutaneous gastrostomy tubes: an issue for adult patients? Dig Dis Sci 2001; 46: $1827-1832$

${ }^{4}$ Kanie J, Akatsu H, Suzuki Y et al. Mechanism of the development of gastric ulcer after percutaneous endoscopic gastrostomy. Endoscopy 2002; 34: $480-$ 482

${ }^{5}$ Chiu PWY, Lam CYW, Lee SW et al. Effect of scheduled second therapeutic endoscopy on peptic ulcer rebleeding: a prospective randomized trial. Gut 2003; 52: $1403-1407$
Corresponding Author

\section{Y. Choi}

Dept. of Surgery

United Christian

Hospital

130 Hip Wo Street, Kwun Tong, Kowloon Hong Kong SAR

China

Fax: $\quad+852-27724566$

E-mail: choichiyee@yahoo.com.hk 